\title{
Rough surface reconstruction at grazing angles by an iterated marching method
}

\author{
Yuxuan Chen ${ }^{1}$ And Mark Spivack ${ }^{2, *}$ \\ ${ }^{1}$ Department of Engineering, University of Cambridge, CB2 1PZ, UK \\ ${ }^{2}$ Department of Applied Mathematics \& Theoretical Physics, University of Cambridge, CB3 OWA, UK \\ *Corresponding author: ms100@cam.ac.uk
}

Compiled February 8, 2018

\begin{abstract}
An iterated marching method is presented for reconstruction of rough perfectly reflecting 1-dimensional surfaces from scattered data arising from a scalar wave at grazing incidence. This is based on coupled integral equations adapted from an earlier approach using the parabolic equation, relating the scattered field at a plane to the unknown surface. Taking the flat surface as an initial guess, these are solved here using at most three iterations. The method is applied to scattered field data generated from the full Helmholtz equations. This approach improves stability and self-consistency. The reconstructed surface profiles are found to be in good agreement with the exact forms. The sensitivity with respect to random noise is also investigated, and the algorithm is found to exhibit a type of self-regularization. (๑) 2018 Optical Society of America
\end{abstract}

OCIS codes: (290.3200) Inverse scattering; (290.5880) Scattering, rough surfaces; (100.3190) Inverse problems.

http://dx.doi.org/10.1364/ao.XX.XXXXXX

\section{INTRODUCTION}

Wave scattering from rough surfaces is a key feature in a wide variety of applications [1-5], and a major area of interest is the inverse problem of reconstructing surface profiles from scattered data [6-19]. Work has also been successfully conducted in the context of sea surface profile retrieval at grazing angles [20] and other regimes [21-23], by use of Doppler or backscattered multiple frequency radar measurements. Typical quantities of interest in that situation include sea state, wave spectra, mean height and statistical parameters. For scalar wave fields with arbitrary incident angles the scattered field obeys the Helmholtz boundary integral equation $[1,24]$. However, when the incident angle is small and most energy is forward-scattered, the propagating wave fields are well described by the parabolic equation [25]. Applying this to the governing Green's function allows the Helmholtz integral equations to be replaced by the parabolic integral equation method [26, 27].

In previous work $[11,12,14,15]$ numerical techniques were derived allowing surface reconstruction in the grazing angle regime. These direct (non-iterative) methods cast the problem as coupled Volterra equations relating the scattered data to the surface fields. This yields a fast 'marching' method, whereby the surface is reconstructed sequentially from a single estimated value at the leading edge of the computational domain. However instability and high sensitivity to the initial ad hoc 'guess' occur in certain cases, and ill-posedness with respect to noise was not explored. This is partly because that algorithm involved ratios of small values, at locations where the incident field amplitude is low and from which only limited information is carried into the scattered field. Furthermore reconstruction was based on scattered data generated via the parabolic integral equations, which neglects wide-angle includuing backscatter.

In this paper, we develop a new approach to the inverse problem for scattering at grazing incidence on a one-dimensional random rough surface. The approach is related to that of [14] but re-derives equations to provide a more straightforward algorithm, coupled with an iterative improvement using a small number of steps (typically two or three). This circumvents the need for an ad hoc initial guess and addresses aspects of the ill-posedness. (Fully iterative approaches are of course used throughout the inverse problems literature, and authors such as $[10,13]$ have successfully applied iterative schemes to rough surface scattering. The current method is strictly speaking a multi-step rather than iterative approach.) The error of the recovered surface is measured with respect to the mesh, surface type, and the location of scattered data measurements.

To provide scattered field data, a randomly rough surface $h(x)$ is generated and the scattered field is obtained along a plane at a given distance from the mean surface. This scattered data is then used in the inversion algorithm to recapture the surface. The main procedures for both direct and inverse problems are based on integral equation formulations. In the direct problem, the scattered field is obtained from the Helmholtz equa- 
tion. In the inverse method, the surface derivative $\partial \Psi / \partial z$ of the (reduced) field $\Psi$ is related to the surface and to the scattered field using the parabolic integral equations. It follows that the surface height can be obtained from another integral equation by substituting $\partial \Psi / \partial z$. Successive improvement is employed here to improve the stability of the method and generate more accurate results. The solution is implemented numerically and several results are presented in which random rough surfaces having different statistical characteristics are recaptured.

\section{MATHEMATICAL FORMULATION}

The details of the Helmholtz (e.g. [1, 24]) and parabolic integral equation methods are given elsewhere $[26,27]$ but for convenience we review them here. Let coordinate axes be $x$ and $z$, where $x$ is the horizontal, $0 \leq x \leq L$, and $z$ is the vertical. Consider a 2-dimensional time harmonic scalar wave $E(x, z)$, resulting from scattering by a rough surface $h(x)$ of a field incident at low grazing angle. We assume the Dirichlet boundary condition. The governing equation for the scattered field is the Helmholtz equation,

$$
\nabla^{2} E(x, z)+k^{2} E(x, z)=f(x, z)
$$

where $k$ is the wavenumber and $f$ is the source term. Denote the incident and scattered components to be $E_{i}$ and $E_{s}$, and the total field $E=E_{i}+E_{s}$. The Kirchoff-Helmholtz equation gives rise to a pair of coupled integral equations

$$
E_{i}(\mathbf{r})=\int_{0}^{L} G_{h}\left(\mathbf{r} ; \mathbf{r}^{\prime}\right) \frac{\partial E}{\partial n}\left(\mathbf{r}^{\prime}\right) d s^{\prime},
$$

where $\mathbf{r}=(x, h(x))$ and $\mathbf{r}^{\prime}=\left(x^{\prime}, h\left(x^{\prime}\right)\right)$ lie on the surface, and

$$
E_{s}(\mathbf{r})=-\int_{0}^{L} G_{h}\left(\mathbf{r} ; \mathbf{r}^{\prime}\right) \frac{\partial E}{\partial n}\left(\mathbf{r}^{\prime}\right) d s^{\prime}
$$

where $\mathbf{r}^{\prime}=\left(x^{\prime}, h\left(x^{\prime}\right)\right)$ still lie on the surface, but $\mathbf{r}=(x, z)$ can be any point in the domain. $G_{h}\left(\mathbf{r} ; \mathbf{r}^{\prime}\right)$ corresponds to the Green's function of the Helmholtz equation which is given by

$$
G_{h}\left(\mathbf{r} ; \mathbf{r}^{\prime}\right)=\frac{1}{4 i} H_{0}^{(1)}\left(k\left|\mathbf{r}-\mathbf{r}^{\prime}\right|\right)
$$

where $H_{0}^{(1)}$ is the zeroth order Hankel function of the first kind. If the incident angle is small enough so that $\cos \alpha \cong 1-\frac{1}{2} \sin ^{2} \alpha$, then forward propagation dominates and the wave has a slowly varying part $\psi$, defined by

$$
\psi(x, z)=E(x, z) \exp (-i k x) .
$$

Within this approximation the parabolic wave equation is immediately obtained [25] for the slowly varying component,

$$
\frac{\partial \psi}{\partial x}=\frac{i}{2 k} \frac{\partial^{2} \psi}{\partial z^{2}} .
$$

which holds for any superposition of plane waves travelling at small angles to the horizontal. Denote the incident and scattered components to be $\psi_{i}$ and $\psi_{s}$, so that $\psi=\psi_{i}+\psi_{s}$.

If we assume the Dirichlet boundary condition, this corresponds to a tranverse electric (TE) field impinging on a perfectly conducting corrugated surface, or acoustic scattering from a pressure release surface. The parabolic equation can then be substituted into the Kirchhoff-Helmholtz equation, which also results in a pair of coupled integrals.

$$
\psi_{i}(\mathbf{r})=-\int_{0}^{x} \frac{\partial \psi\left(\mathbf{r}^{\prime}\right)}{\partial z} G_{p}\left(\mathbf{r} ; \mathbf{r}^{\prime}\right) d x^{\prime}
$$

where $\mathbf{r}=(x, h(x))$ and $\mathbf{r}^{\prime}=\left(x^{\prime}, h\left(x^{\prime}\right)\right)$ lie on the surface, and

$$
\psi_{s}(\mathbf{r})=\int_{0}^{x} \frac{\partial \psi\left(\mathbf{r}^{\prime}\right)}{\partial z} G_{p}\left(\mathbf{r} ; \mathbf{r}^{\prime}\right) d x^{\prime}
$$

where $\mathbf{r}^{\prime}=\left(x^{\prime}, h\left(x^{\prime}\right)\right)$ still lie on the surface, but $\mathbf{r}=(x, z)$ can be any point in the domain. $G_{p}\left(\mathbf{r} ; \mathbf{r}^{\prime}\right)$ corresponds to the Green's function of the parabolic wave equation which is given by

$$
G_{p}\left(\mathbf{r}, \mathbf{r}^{\prime}\right)=\left\{\begin{array}{l}
\frac{1}{2}\left[\frac{i}{2 \pi k\left(x-x^{\prime}\right)}\right]^{1 / 2} \exp \left[\frac{i k\left(z-z^{\prime}\right)^{2}}{2\left(x-x^{\prime}\right)}\right], \quad \mathbf{x}^{\prime}<x \\
0, \quad \text { otherwise }
\end{array} .\right.
$$

The surface derivatives $\partial E / \partial n$ and $\partial \psi / \partial n$ along the surface $h(x)$ are consideredf as functions of $x$, and will be denoted by $E^{\prime}$ and $\psi^{\prime}$ respectively. We will denote the constant $\alpha=1 / 2(i / 2 \pi k)^{1 / 2}$.

The incident field or the source condition is taken to be a Gaussian beam travelling horizontally,

$$
\psi_{i}(x, z)=\frac{w}{\left(w^{2}+2 i x / k\right)^{1 / 2}} \exp \left[-\frac{\left(z-z_{0}\right)^{2}}{w^{2}+2 i x / k}\right],
$$

where $w$ is the initial width. Thus the source is centered at $\mathbf{r}=\left(0, z_{0}\right)$, which is assumed to be well separated from the rough surface so that the incident field amplitude on the surface near $x=0$ is negligibly small. As $x$ increases this rises to a peak and then decays as $1 / \sqrt{x}$.

\section{GENERATION OF SCATTERED DATA}

In order to generate scattered data measurements, a random statistically stationary rough surface $h(x)$ is first chosen with a given autocorrelation function $\rho(\eta)$ where $\eta$ is spatial separation. In practice this may be simulated by summation of Fourier modes with independent random phases chosen uniformly in $[0,2 \pi)$ and a filter function depending on $\rho$. (Far more computationally efficient methods are available but these are not needed here.)

For the surface reconstructions we will compare results using data generated both by the full Helmholtz equation, and by the parabolic equation approximation (which excludes backscatter). This is done in order to examine the extent to which the results depend on the PE model assumptions underlying the inversion algorithm.

The integration domain $[0, L]$ is discretized uniformly by $\mathrm{N}$ points with $x_{l}$ for $l=0,1, \cdots, N$, where $x_{0}=0$ and $x_{N}=$ $L$. Denote the space of each subinterval $\delta=x_{l+1}-x_{l}$. The procedures for the parabolic case are similar to those given in $[14,26]$ will not be repeated in full detail here.

\section{A. Calculation of surface derivative}

For any point $x_{n} \in\left[x_{1}, x_{N}\right]$, equation Eq. (2) can be divided into $N$ subintervals and be written as a summation over $\mathrm{N}$ terms,

$$
E_{i}\left(x_{n}, h\left(x_{n}\right)\right)=\sum_{l=1}^{N} \int_{x_{l-1}}^{x_{l}} G_{h}\left(x_{n}, h\left(x_{n}\right) ; x^{\prime}, h\left(x^{\prime}\right)\right) E^{\prime}\left(x^{\prime}\right) d s^{\prime}
$$

Assume that $E^{\prime}$ varies very slowly over each subinterval compared to the Green's function. Then, $E^{\prime}$ can be treated as constant on each subinterval, and these constants can be taken out of the subintegrals using the mid-point rule,

$$
E_{i}\left(x_{n}, h\left(x_{n}\right)\right)=\sum_{l=1}^{N} E^{\prime}\left(X_{l}\right) \int_{x_{l-1}}^{x_{l}} G_{h}\left(x_{n}, h\left(x_{n}\right) ; x^{\prime}, h\left(x^{\prime}\right)\right) d s^{\prime},
$$


where $X_{l}$ is the middle point of each subinterval $\left[x_{l-1}, x_{l}\right]$, i.e. $X_{l}=\frac{1}{2}\left(x_{l-1}+x_{l}\right)$. Taking $n=1,2, \cdots, N$, a linear system of $N$ equations are obtained. Denote two vectors of size $N$ as follows

$$
\begin{aligned}
& \mathbf{E}^{\prime}=\left[E^{\prime}\left(X_{1}\right), E^{\prime}\left(X_{2}\right), \cdots, E^{\prime}\left(X_{N}\right)\right], \\
& \mathbf{E}_{\mathbf{i}}=\left[E_{i}\left(x_{1}, h\left(x_{1}\right)\right), E_{i}\left(x_{2}, h\left(x_{2}\right)\right), \cdots, E_{i}\left(x_{N}, h\left(x_{N}\right)\right)\right] .
\end{aligned}
$$

They are, thus, related by

$$
A_{1} \mathbf{E}^{\prime}=\mathbf{E}_{\mathbf{i}}
$$

where $A_{1}$ is a matrix of size $N \times N$ given by

$$
A_{1}(n, l)=\int_{x_{l-1}}^{x_{l}} G_{h}\left(x_{n}, h\left(x_{n}\right) ; x^{\prime}, h\left(x^{\prime}\right)\right) d s^{\prime} .
$$

To leading order, the surface differential is the same as the line segment. Therefore, we have

$$
\begin{array}{r}
d s^{2} \approx d L^{2}=d x^{2}+\left(h\left(x_{l-1}\right)^{2}-h\left(x_{l}\right)\right)^{2}=\left(1+\frac{d h^{2}}{d x^{2}}\right) d x^{2} \\
\approx\left(1+h^{\prime}(x)^{2}\right) d x^{2} .
\end{array}
$$

The integral in the matrix then becomes

$$
\begin{aligned}
& \int_{x_{l-1}}^{x_{l}} G_{h}\left(x_{n}, h\left(x_{n}\right) ; x^{\prime}, h\left(x^{\prime}\right)\right) d s^{\prime} \\
& \approx \frac{1}{4 i} \int_{x_{l-1}}^{x_{l}} H_{0}^{(1)}\left(k\left|\mathbf{r}_{\mathbf{n}}-\mathbf{r}^{\prime}\right|\right) \sqrt{1+h^{\prime}\left(x^{\prime}\right)^{2}} d x^{\prime} .
\end{aligned}
$$

If $l \neq n$ and $l \neq n+1$, we again employ the mid-point rule for $H_{0}^{(1)}$, namely, assume that $H_{0}^{(1)}$ varies slowly in the subinterval and treat it as constant. Thus, we get

$$
A_{1}(n, l)=\frac{\delta}{4 i} H_{0}^{(1)}\left(k\left|\left(x_{n}, h\left(x_{n}\right)\right)-\left(X_{l}, h\left(X_{l}\right)\right)\right|\right) \sigma_{l},
$$

where $\sigma_{l}=\sqrt{1+h^{\prime}\left(X_{l}\right)^{2}}$. On the other hand, If $l=n$ or $l=$ $n+1$, there is a singularity in the integral. The integral can be approximated by

$$
A(n, n)=A(n, n+1)=\frac{1}{k^{2}}\left(\frac{\eta_{0}}{4 i}+\frac{1}{2 \pi} \eta_{0}+\frac{1}{2 \pi} \eta_{0}\left[\ln \left(\frac{\eta_{0}}{2}\right)-1\right]\right),
$$

where $\eta_{0}$ is a constant. The derivation is shown in Appendix 6.

\section{B. Calculation of scattered field data}

The scattered field generated by Eq. (3) can be obtained in the similar way. Note that for equation Eq. (3), $\mathbf{r}=(x, z)$ and $\mathbf{r}^{\prime}=$ $\left(x^{\prime}, h\left(x^{\prime}\right)\right)$. The discretized form for scattered wave becomes

$$
E_{S}\left(x_{n}, z\right)=-\sum_{r=1}^{N} \int_{x_{r-1}}^{x_{r}} G_{h}\left(x_{n}, z ; x^{\prime}, h\left(x^{\prime}\right)\right) E^{\prime}\left(x^{\prime}\right) d s^{\prime}
$$

Again, we treat the surface derivative $E^{\prime}$ as constant on each subintegral and apply the mid-point rule,

$$
E_{S}\left(x_{n}, z\right)=-\sum_{r=1}^{N} E^{\prime}\left(X_{r}\right) \int_{x_{r-1}}^{x_{r}} G_{h}\left(x_{n}, z ; x^{\prime}, h\left(x^{\prime}\right)\right) d s^{\prime} .
$$

We denote the vector of scattered field at height $z$ of size $N$,

$$
\mathbf{E}_{\mathbf{s}}=\left[E_{S}\left(x_{1}, z\right), E_{s}\left(x_{2}, z\right), \cdots, E_{s}\left(x_{N}, z\right)\right] .
$$

A linear system containing the scattered field vector is then obtained

$$
\mathbf{E}_{\mathbf{s}}=A_{2} \mathbf{E}^{\prime}
$$

where $A_{2}$ is a matrix of size $N \times N$ given by

$$
A_{2}(n, r)=\int_{x_{r-1}}^{x_{r}} G_{h}\left(x_{n}, z ; x^{\prime}, h\left(x^{\prime}\right)\right) d s^{\prime} .
$$

We assume the scattered data is available at some non-zero distance $z$ from the surface. Therefore, there is no singularity in the integral. Apply the equation Eq. (12), the entry of matrix becomes

$$
A_{2}(n, r)=-\frac{\delta}{4 i} H_{0}^{(1)}\left(k\left|\left(x_{n}, h\left(x_{n}\right)\right)-\left(X_{r}, h\left(X_{r}\right)\right)\right|\right) \sigma_{r} .
$$

\section{INVERSE PROBLEM}

In the problem to be treated here the surface $h(x)$ (along with $\psi^{\prime}$ ) is unknown, and we assume we have complex-valued scattered field data along a plane parallel to, and at known distance from, the mean surface plane. The treatment for the surface inverse problem here is adapted broadly from that of [14] but is intended to address issues of stability, consistency, and the dependence on an initial ad hoc guess. The question of consistency arises from the use of two equations Eq. (6) and Eq. (7) with different approximations to recover $\psi^{\prime}$. This can potentially give rise to inaccuracy. The problem of stability is related to the equation used in [14] to recover the surface:

$$
h=\left[\frac{-\alpha \int_{0}^{x}\left(x-x^{\prime}\right)^{1 / 2} \psi^{\prime}\left(x^{\prime}\right)}{\psi_{i}(x, 0)}-1\right]\left(\frac{w^{2}+\frac{2 i x}{k}}{2 z_{0}}\right),
$$

Solution is based on division by the incident field $\psi_{i}(x, 0)$ successively starting from small values of $x$, where this field is nearly zero. Both these potential difficulties in effect result from the simultaneous recovery of $h(x)$ and $\psi^{\prime}(x)$. The method here circumvents this by separating this into two parts where $\psi^{\prime}$ is produced first and then $h(x)$ is reconstructed by Eq. (7).

Some general remarks can be made here:

1. The assumption of scattered data along a plane parallel to the surface is a convenience, which greatly simplifies the algorithm, but is not fundamental to it. On the other hand the grazing illumination of the surface is a key property. Grazing angles often feature automatically (e.g. [28-31] and above-mentioned references), but the possibility of using a suitably oriented incident field is not restricted to those cases.

2. The approach assumes perfectly conducting boundary conditions, but is not inherently restricted to the Dirichlet condition (TE incidence) or to scalar waves for which it is formulated here. It is also important to consider the eventual aim to treat to 3dimensional configurations. The current approach is in principle capable of this (as discussed in 6) in part due to developments in fast boundary integral methods for low grazing angles. Permeable boundaries between half-spaces are beyond the scope of the current study although there is some prospect of treating rough coatings overlaying perfectly conducting substrates.

\section{A. Recovery of surface derivative}

The domain $[0, L]$ is discretized similarly to the direct problem, whereas, the upper limit of the integration becomes $x_{n}$ for parabolic equation. For any point $x_{n} \in\left[x_{1}, x_{N}\right]$, equation Eq. (6) can be divided into $n$ subintegrals and be written as a summation over $n$ subintervals,

$$
\begin{aligned}
\psi_{i}\left(\mathbf{r}_{\mathbf{n}}\right) & =-\sum_{l=1}^{n} \int_{x_{l-1}}^{x_{l}} G_{p}\left(\mathbf{r} ; \mathbf{r}^{\prime}\right) \psi^{\prime}\left(\mathbf{r}^{\prime}\right) d x^{\prime} \\
& =-\sum_{l=1}^{n} \int_{x_{l-1}}^{x_{l}} \alpha \frac{1}{\sqrt{x_{n}-x^{\prime}}} \exp \left[\frac{i k\left(z_{n}-z^{\prime}\right)^{2}}{2\left(x_{n}-x^{\prime}\right)}\right] \psi^{\prime}\left(\mathbf{r}^{\prime}\right) d x^{\prime}
\end{aligned}
$$


where $\mathbf{r}_{\mathbf{n}}=\left(x_{n}, h\left(x_{n}\right)\right), z_{n}=h\left(x_{n}\right)$ and $z^{\prime}=h\left(x^{\prime}\right)$. The initial goal is to find the discrete $\psi^{\prime}$. We may assume that $\psi^{\prime}$ and the exponential term vary slowly over each interval compared to $1 / \sqrt{x_{n}-x^{\prime}}$. For $l<n$, the integral has no singularity. Thus $\psi^{\prime}$ and the exponential term can be treated as constant on each subinterval, then these constants can be taken out of the subintegrals.

$$
\begin{aligned}
& \psi_{i}\left(x_{n}, h\left(x_{n}\right)\right) \approx \\
& -\sum_{l=1}^{n-1} \alpha \exp \left[\frac{i k\left(h\left(x_{n}\right)-h\left(X_{l}\right)\right)^{2}}{2\left(x_{n}-X_{l}\right)}\right] \psi^{\prime}\left(X_{l}\right) \int_{x_{l-1}}^{x_{l}} \frac{d x^{\prime}}{\sqrt{x_{n}-x^{\prime}}} \\
& -\psi^{\prime}\left(X_{n}\right) \int_{x_{n-1}}^{x_{n}} \alpha \exp \left[\frac{i k\left(h\left(x_{n}\right)-h\left(x^{\prime}\right)\right)^{2}}{2\left(x_{n}-x^{\prime}\right)}\right] \frac{d x^{\prime}}{\sqrt{x_{n}-x^{\prime}}}
\end{aligned}
$$

where $X_{l}=\frac{1}{2}\left(x_{l-1}+x_{l}\right)$. The last subintegral with singularity can be approximated by

$$
2 \frac{\alpha}{\beta}\left[C\left(Z_{0}\right)+i S\left(Z_{0}\right)\right]
$$

where $Z_{0}$ and $\beta$ are constants defined in Appendix 6 and $C$ and $S$ are the Fresnel integrals derived similarly to [27], as is also shown in Appendix 6. The unknown surface $h$ is still contained implicitly in the formulation Eq. (20). The starting point is to have an initial guess for $h$. Then everything turns to be known in Eq. (20) except the discrete $\psi^{\prime}\left(X_{l}\right)$. The surface derivative $\psi^{\prime}$ can be approximated by a linear system,

$$
B \Psi^{\prime}=\Psi_{i}
$$

where

$$
\begin{aligned}
B(n, l) & =\left\{\begin{array}{l}
-\alpha \exp \left[\frac{i k\left(h\left(x_{n}\right)-h\left(X_{l}\right)\right)^{2}}{2\left(x_{n}-X_{l}\right)}\right] \int_{x_{l-1}}^{x_{l}} \frac{d x^{\prime}}{\sqrt{x_{n}-x^{\prime}}}, \quad l<n \\
2 \frac{\alpha}{\beta}\left[C\left(Z_{0}\right)+i S\left(Z_{0}\right)\right], \quad l=n
\end{array}\right. \\
\Psi^{\prime} & =\left[\psi^{\prime}\left(X_{1}\right), \psi^{\prime}\left(X_{2}\right), \cdots, \psi^{\prime}\left(X_{N}\right)\right] \\
\Psi_{i} & =\left[\psi_{i}\left(x_{1}, h\left(x_{1}\right)\right), \psi_{i}\left(x_{2}, h\left(x_{2}\right)\right), \cdots, \psi_{i}\left(x_{N}, h\left(x_{N}\right)\right)\right] .
\end{aligned}
$$

for $1 \leq l \leq n \leq N$. The key property is that $B$ is lower triangular and its inversion is highly efficient computationally.

\section{B. Surface reconstruction}

Suppose now that $\psi_{s}\left(x_{n}, z\right)$ is known at each point $x_{n} \in\left[x_{1}, x_{N}\right]$ along a plane at fixed distance $z$. The difficulty to find $h$ from Eq. (7) is that $h(x)$ is inside the exponential term in the Green's function $G_{p}$. Equation Eq. (7) is again a summation of subintegrals,

$$
\begin{aligned}
\psi_{S}\left(x_{n}, z\right) & =\sum_{r=1}^{n} \int_{x_{r-1}}^{x_{r}} G\left(\mathbf{r}, \mathbf{r}^{\prime}\right) \psi^{\prime}\left(\mathbf{r}^{\prime}\right) d x^{\prime} \\
& =\sum_{r=1}^{n} \int_{x_{r-1}}^{x_{r}} \alpha \frac{1}{\sqrt{x_{n}-x^{\prime}}} \exp \left[\frac{i k\left(z-h\left(x^{\prime}\right)\right)^{2}}{2\left(x_{n}-x^{\prime}\right)}\right] \psi^{\prime}\left(\mathbf{r}^{\prime}\right) d x^{\prime}
\end{aligned}
$$

Under the same assumption as in section A $\psi^{\prime}$ and the exponential term are treated as constants in each subinterval, and the formula then becomes

$\psi_{s}\left(x_{n}, z\right)=\sum_{r=1}^{n} \alpha \exp \left[\frac{i k\left(z-h\left(X_{r}\right)\right)^{2}}{2\left(x_{n}-X_{r}\right)}\right] \psi^{\prime}\left(X_{r}\right) \int_{x_{r-1}}^{x_{r}} \frac{1}{\sqrt{x_{n}-x^{\prime}}} d x^{\prime}$,

where $X_{r}=\frac{1}{2}\left(x_{r-1}+x_{r}\right)$. The surface derivative $\psi^{\prime}$ generated by Eq. (22) can be substituted in here. The unknown $h$ can now be calculated at successive points by

$$
\begin{gathered}
\exp \left[\frac{i k\left(z-h\left(X_{n}\right)\right)^{2}}{2\left(x_{n}-X_{n}\right)}\right]=\left[\alpha \psi^{\prime}\left(X_{n}\right) \int_{x_{n-1}}^{x_{n}} \frac{d x^{\prime}}{\sqrt{x_{n}-x^{\prime}}}\right]^{-1} \\
\times\left(\psi_{s}-\sum_{r=1}^{n-1} \alpha \exp \left[\frac{i k\left(z-h\left(X_{r}\right)\right)^{2}}{2\left(x_{n}-X_{r}\right)}\right] \psi^{\prime}\left(X_{r}\right) \int_{x_{r-1}}^{x_{r}} \frac{1}{\sqrt{x_{n}-x^{\prime}}} d x^{\prime}\right) .
\end{gathered}
$$

The problem is now reduced to that of solving the exponential equation of $h$. If taking the direct logarithm, the exponential term with complex variables may be ambiguous since $\log z=$ $\ln (|z|)+i \arg (z)$, where $\arg (z)=\operatorname{Arg}(z)+2 k \pi, \operatorname{Arg}(z) \in$ $[-\pi, \pi], k \in \mathbb{Z}$. However in the cases treated here $h$ is sufficiently small to simply take $\operatorname{Arg}(z)$ in the imaginary part, i.e.

$$
\operatorname{Ln}(z)=\ln |z|+i \operatorname{Arg}(z)
$$

Therefore, the surface height can be reconstructed from

$$
\begin{gathered}
h\left(X_{n}\right)=z-\sqrt{\frac{2}{i k}\left(x_{n}-X_{n}\right) \operatorname{Ln}\left(S_{n}\right)} \\
S_{n}=\frac{\psi_{s}\left(x_{n}, z\right)-\sum_{r=1}^{n-1} \alpha \exp \left[\frac{i k\left(z-h\left(X_{r}\right)\right)^{2}}{2\left(x_{n}-X_{r}\right)}\right] \psi^{\prime}\left(X_{r}\right) \int_{x_{r-1}}^{x_{r}} \frac{1}{\sqrt{x_{n}-x^{\prime}}} d x^{\prime}}{\alpha \psi^{\prime}\left(X_{n}\right) \int_{x_{n-1}}^{x_{n}} \frac{d x^{\prime}}{\sqrt{x_{n}-x^{\prime}}}}
\end{gathered}
$$

Note that the results shown here are derived for surface heights smaller than a wavelength. This still entails multiple scattering, but larger surface heights give rise to greater phase variations along the surface, which require careful 'unwrapping' in order to resolve ambiguities. It is possible to apply this treatment successfully to larger surface heights in this way, but this is not necessary to demonstrate the principles here. In applying this to increasing surface heights, the key criterion is whether scattering remains well-described by the parabolic equation. That depends critically on the horizontal roughness scale, as well as the vertical. Despite the enormous literature on the parabolic equation the breakdown of the PE for rough surfaces has not yet been well categorized.

Successive improvement can be employed to reconstruct the surface more accurately, using a small number of iterations (typically three). For finding $\psi^{\prime}$, the starting point is to assume $h(x)=0$. Then $\psi^{\prime}$ can be obtained without using any scattered data, which leads to ill-posedness of the problem. In order to reuse Eq. (6) by scattered data, let $h_{1}=0$ be the initial guess. After $\Psi^{\prime}$ is obtained from the first part, then a new surface height $h_{2}$ is constructed by the second part. Then this process is repeated. A new set of $\Psi^{\prime}$ can be found by substituting $h_{2}$ in the first part. The new surface height $h_{3}$ is then generated. This kind of iteration can be repeated several times to get a satisfactory surface reconstruction. The whole procedure for the inverse problem is shown in Algorithm 1.

Algorithm 1. Reconstruction of the surface height $h(x)$

1: Input: $v$ : number of iterations, $\psi_{i}(x, z), \psi_{s}\left(x_{i}, z\right): i=$ $1,2, \cdots, N$

2: Set $h=0$ as initial guess

3: for $j=1, \cdots, v$ do

4: $\quad$ Generate $\Psi^{\prime}$ from $B \Psi^{\prime}=\Psi_{i}$ constructed by Eq. (23) using the iterative $h$

5: $\quad$ Reconstruct $h\left(X_{i}\right)$ from Eq. (25) using $\Psi^{\prime}$ obtained above 


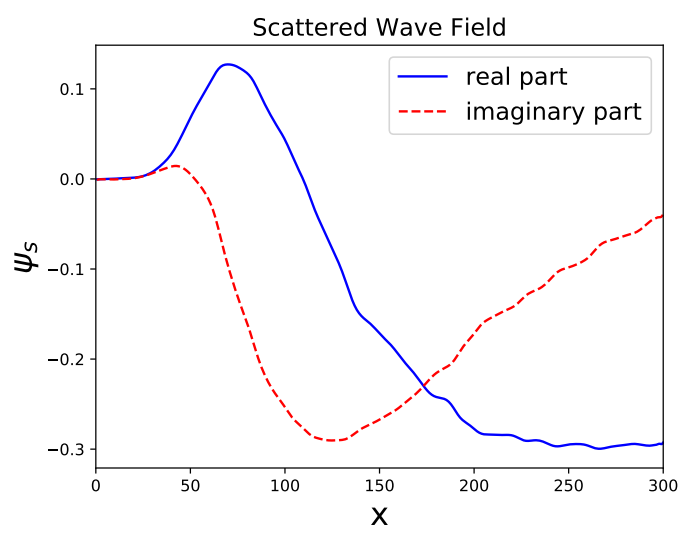

Fig. 1. Scattered field data (real and imaginary components) generated by Helmholtz equation for a Gaussian autocorrelation functions.

\section{RESULTS}

All procedures here have been implemented in Python. Throughout these calculations all functions have been treated as complexvalued. This is a computational convenience; the resulting surface reconstruction may acquire a small non-physical imaginary component which we can neglect.

For these numerical experiments the random rough surface is chosen to have a given autocorrelation function (a.c.f.) $\rho(\eta)$, where $\eta=x^{\prime}-x$. Two such examples are taken here. The first has a 'sub-fractal' a.c.f. with the form

$$
\rho(\eta)=<h(x) h(x+\eta)>=\sigma^{2}\left(1+\frac{|\eta|}{l}\right) \exp \left(-\frac{|\eta|}{l}\right),
$$

where $l=8$ is the wavelength and $\rho(0)=\sigma^{2}$ is the variance (so that $\sigma$ is the rms surface height and is a measure of surface roughness). This a relatively jagged surface with peaks on a small scale. The second surface is smoother at small scales, given by a Gaussian a.c.f.:

$$
\rho(\eta)=\sigma^{2} \exp \left(-\frac{\eta^{2}}{l^{2}}\right) .
$$

Both examples use the wave number $k=1$, incident Gaussian beam centred at $z_{0}=22.4$ at zero grazing angle, and initial width $w=8$. The number of grid points $N$ along the surface was taken to be about 500 in the tests here. The typical peak-totrough of the surfaces was around 0.4. The scattered wave field was sampled along a plane at the height $z=0.7$. The scattered wave fields generated by Helmholtz equations, e.g. Eq. (10) and Eq. (15), is shown in Figure 1. The surface is recovered using scattered field data generated by both full Helmholtz equation and parabolic equation models, in order partly to measure the effect on solutions of the model assumptions. First the scattered data is generated by parabolic equation [27]. The reconstructed surface $h$ is plotted against the original surface heights shown in Figures 2 and 3. The reconstructions are significantly improved compared to previous results $[14,15]$. Second, we use Helmholtz equation to generate the scattered field. The surface reconstructions are shown in Figures 4 and 5.

The approximations work well for both cases, and the solution recaptures most of the detailed features of original surface. This validates the performance of the approach. The surface reconstruction appears more accurate in the case of the smoother
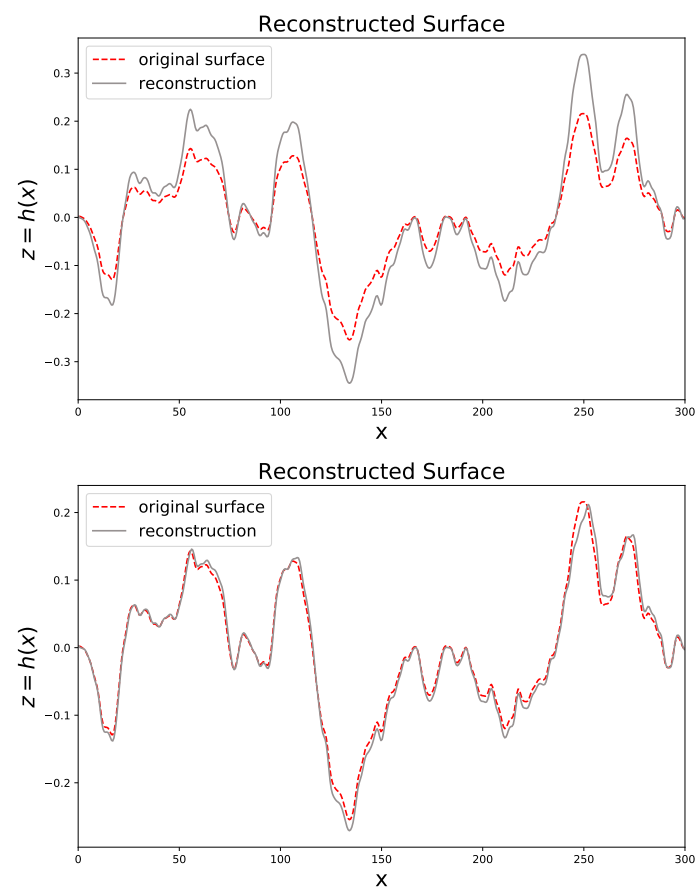

Fig. 2. Plots of sub fractal surface $h$ from inverse problem with parabolic scattered data, for first iteration (upper) and third iteration (lower).

Gaussian autocorrelation function. This is unsurprising: At the moderate spatial resolutions used here the more jagged surface features are harder to approximate. However, by taking several iterations, both types of surface fit well to the origin surfaces as the approximation of $\psi^{\prime}$ improves. As might be expected, surface reconstructions based on the purely forward-scattered data from the parabolic equation converge more readily. By comparison, the initial (left-most) region of the reconstructed surface exhibits oscillatory behaviour when using the Helmholtz scattered data. However, the reconstruction tends to stabilize as it progresses to the right.

We also briefly consider the sensitivity of solutions to random white noise added to the scattered data, and give some illustrative results. The applied noise levels ranged from around $1 \%$ to $5 \%$ of the averaged scattered field amplitude. More specifically, independent random complex values $\epsilon_{r}+i \epsilon_{i}$ were added to each data point, drawn uniformly from an interval $[-\Sigma, \Sigma]$ for a range of values of $\Sigma$. Crucially, this noise was added throughout the spatial domain, including initial regions where the original data was near zero; this has a greatly disproportionate effect on the results locally. The resulting reconstruction shows some oscillations. However these oscillations are qualitatively similar to the noise itself and effectively can be filtered out after reconstruction if we assume the surface is smooth on the smallest scale size of the grid. Comparison between actual and filtered reconstructed surfaces for $1 \%$ and $3 \%$ noise added to two scattered data are shown in Figure 6 and 7. These are at the third iteration and the filtration is done by a simple five point moving average. Note that the initial region oscillation in Helmholtz scattered data case is still caused by the approximation of parabolic equation. This appears to be a type of self-regularization and a property of the underlying marching method for solving the coupled integral equations, rather than of the iterative element of the algorithm.

The error of the reconstructed surface can be directly visu- 

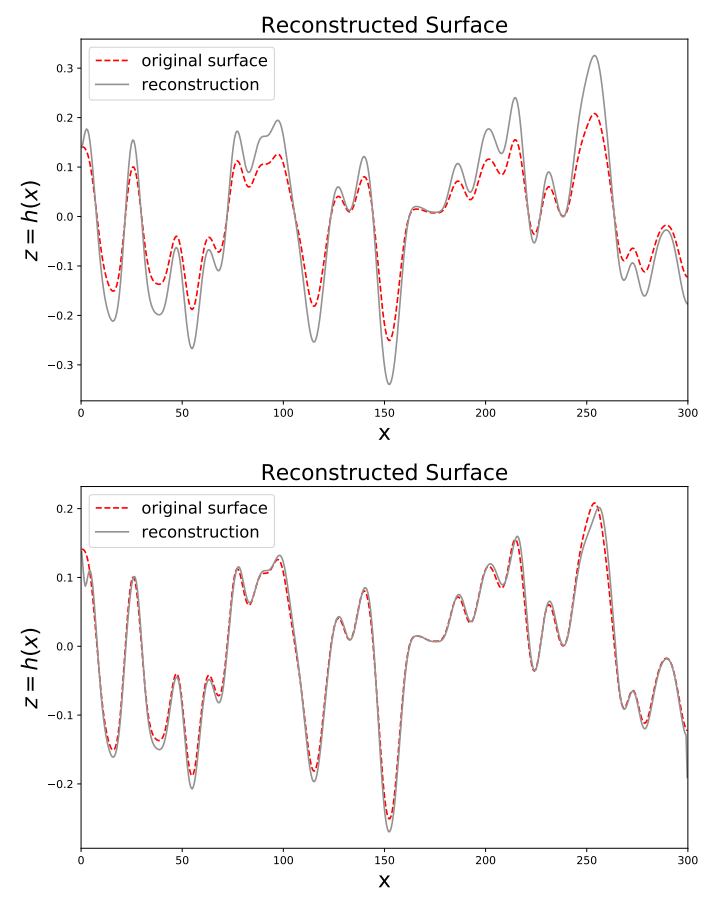

Fig. 3. Plots of Gaussian type surface $h$ from inverse problem with parabolic scattered data, for first iteration (upper) and third iteration (lower).
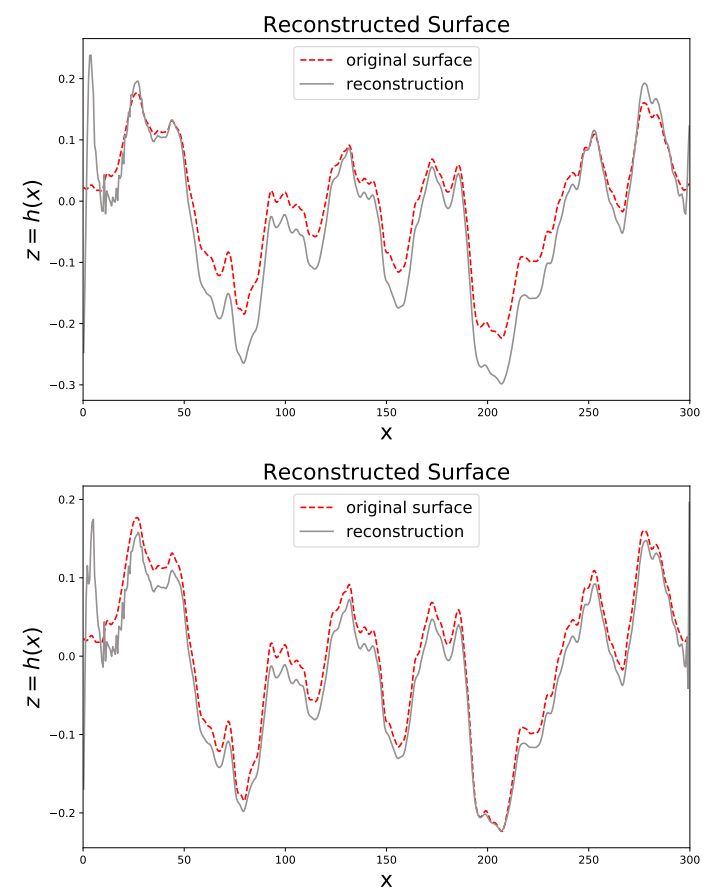

Fig. 4. Plots of sub fractal surface $h$ from inverse problem with Helmholtz scattered data, for first iteration (upper) and third iteration (lower).
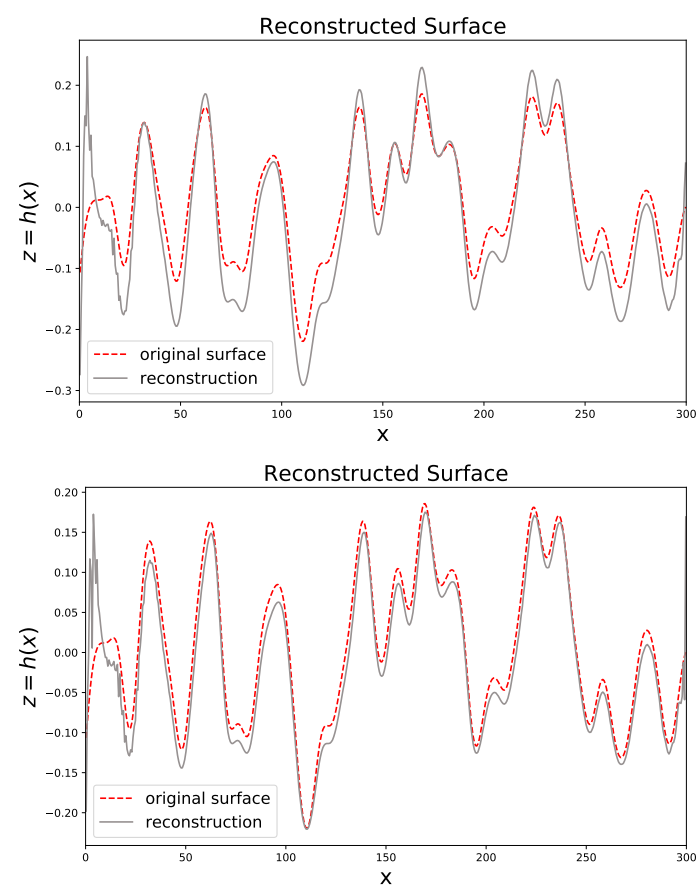

Fig. 5. Plots of Gaussian type surface $h$ from inverse problem with Helmholtz scattered data, for first iteration (upper) and third iteration (lower).


Fig. 6. Comparison of actual surface reconstruction (upper) with filtered reconstruction (lower) at third iteration for noise levels of around 1\% added to Helmholtz scattered data. 

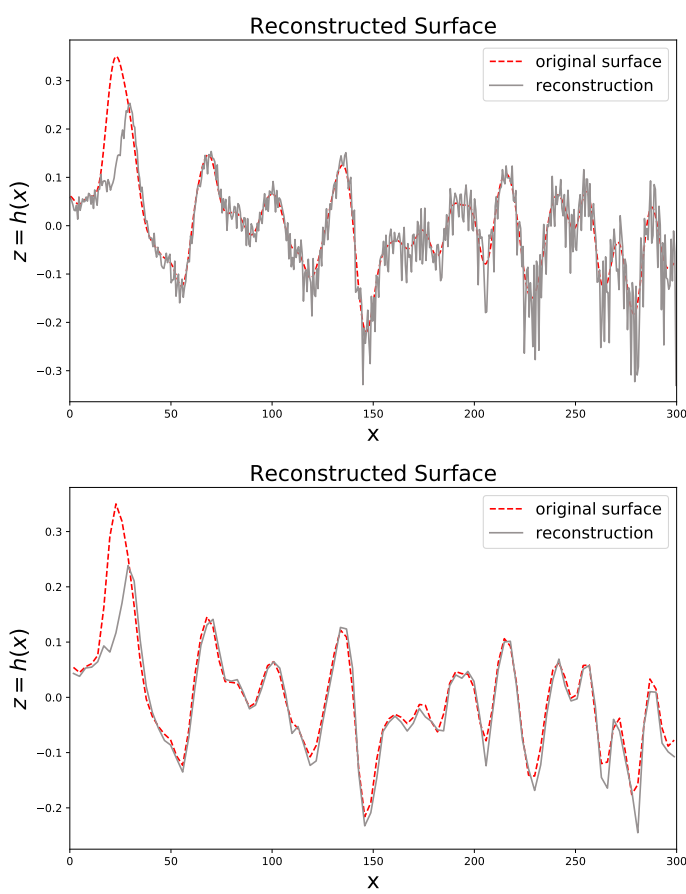

Fig. 7. Comparison of actual surface (upper) with filtered reconstruction (lower) at third iteration for noise levels of around $3 \%$ added to parabolic scattered data.

alized in the plots. To be more accurate, we perform an error analysis by using $L_{2}$ norm of the residual,

$$
e=\left(\sum_{i=1}^{N}\left(h_{i}-H_{i}\right)^{2}\right)^{1 / 2},
$$

where $h_{i}$ and $H_{i}$ are approximated and original surfaces. We first plot the average distance error

$$
\frac{\left(\sum_{i=1}^{j}\left(h_{i}-H_{i}\right)^{2}\right)^{1 / 2}}{X_{j}}
$$

which is shown in Figure 8 with logarithm size. It appears that the error is raised at the initial region due to the highly oscillatory part of the reconstruction, then drops quickly as the solution is stabilized. We also choose some controlling parameters, which are surface type, number of nodes, and height of scattered data generated, to characterize the average node error which is defined as

$$
\frac{\left(\sum_{i=1}^{N}\left(h_{i}-H_{i}\right)^{2}\right)^{1 / 2}}{N} .
$$

Table 1 gives the error with respect to different numbers of nodes and surface type. As the number of nodes increases, it is apparent that the average error per node drops. Errors for different locations of scattered data measurements are shown in Table 2. It is clear that the scattered data at height 0.7 gives the best reconstruction. In all of tests, the errors for both surface types are similar, although as mentioned above the reconstruction for Gaussian surface is slightly better at this resolution.

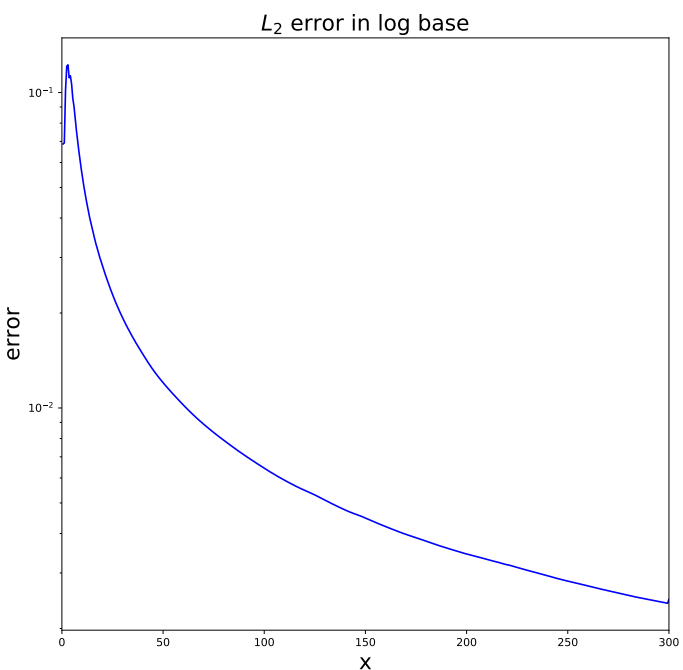

Fig. 8. Logarithmic plot of error as a function of distance

\section{CONCLUSIONS}

The inverse problem of reconstructing an unknown rough surface from forward scattered data at low grazing angles is examined. The rough surface is recaptured via solution of a pair of coupled parabolic (Volterra) integral equations relating the surface height $h$ and the surface derivative of wave field $\psi^{\prime}$ to the scattered field at a fixed distance into the medium. The solutions are then iterated a small number of times, typically three (so this may be regarded as a three-step method). Results are obtained based on wide-angle scattered data obtained from the full Helmholtz equation and (for comparison) forward-scattered data from the parabolic approximation. The method here is based partly on a previous non-iterative (single-step) method [14], but substantially adapted to address issues of stability and consistency. One drawback of the original approach was that it required an initial guess of surface values in a region in which surface illumination/insonification may be low, which worsens the ill-posedness of the problem. The present method overcomes these issues via the introduction of a small number of iterations, and by recasting the equations to decouple the recovery of unknown surface height and surface field.

Close agreement is found between reconstructed and original surfaces. Each iterative step is highly efficient computationally as it requires only the inversion of a lower triangular system. The approach is equivalent to 'marching' the solution along the direction of propagation. This appears to leads to a type of spatial self-regularization, in which errors near the leading edge become suppressed as the marching scheme proceeds. This aspect merits further analytical investigation.

We have also examined the effect of adding random measurement noise. This gives rise to surface reconstructions which closely follow the exact form, plus an additional rapidly-varying component. This perturbation is amenable to filtering, and after this is carried out the resulting surface reconstructions agree well with the original forms.

For perfectly conducting surfaces the principles of this approach appear well-suited to other regimes in which forward scattering dominates. Work is currently underway in the extension to the Neumann boundary condition (TM incidence) and on ducting due to a refractive index profile. The latter leads to increased multiple scattering and is possible only where the 


\begin{tabular}{|c|c|c|c|c|}
\hline & \multicolumn{2}{|c|}{ Parabolic scattered data } & \multicolumn{2}{c|}{ Helmholtz scattered data } \\
\hline Number of nodes & Sub-fractal surface & Gaussian surface & Sub-fractal surface & Gaussian surface \\
\hline 300 & $9.86 \mathrm{E}-04$ & $1.30 \mathrm{E}-03$ & $4.92 \mathrm{E}-03$ & $5.00 \mathrm{E}-03$ \\
\hline 500 & $4.23 \mathrm{E}-04$ & $4.54 \mathrm{E}-04$ & $1.33 \mathrm{E}-03$ & $1.49 \mathrm{E}-03$ \\
\hline 800 & $2.32 \mathrm{E}-04$ & $1.99 \mathrm{E}-04$ & $1.22 \mathrm{E}-03$ & $1.09 \mathrm{E}-03$ \\
\hline
\end{tabular}

Table 1. L2 error per node for different numbers of nodes

\begin{tabular}{|c|c|c|c|c|}
\hline & \multicolumn{2}{|c|}{ Parabolic scattered data } & \multicolumn{2}{c|}{ Helmholtz scattered data } \\
\hline $\begin{array}{c}\text { Height of } \\
\text { scattered data }\end{array}$ & Sub-fractal & Gaussian & Sub-fractal & Gaussian \\
\hline 0.5 & $1.88 \mathrm{E}-03$ & $2.24 \mathrm{E}-03$ & $2.60 \mathrm{E}-03$ & $3.21 \mathrm{E}-03$ \\
\hline 0.7 & $4.85 \mathrm{E}-04$ & $5.42 \mathrm{E}-04$ & $1.33 \mathrm{E}-03$ & $1.46 \mathrm{E}-03$ \\
\hline 1.0 & $2.33 \mathrm{E}-04$ & $3.06 \mathrm{E}-04$ & $3.15 \mathrm{E}-03$ & $3.37 \mathrm{E}-03$ \\
\hline
\end{tabular}

Table 2. L2 error per node for different heights scattered data measured

Green's function is available (see e.g. [32]). In the 3-dimensional case, the presence of significant cross-polarization in the scattered data $[5,28]$ and the greatly increased computational cost present considerable extra challenges, but initial studies suggest that the approach remains feasible. The parabolic equations which provide the crucial computational advantages in 2-dimensions can be replaced in 3-dimensions by the use of operator splitting (see e.g. [31].

On the other hand, even in 2-dimensions, impedance surfaces and transmission problems give rise to higher data requirements, and greater level of ill-posedness which we have not resolved with this approach. With the possible exception of a thin coating on a flat perfect reflector, such situations call for alternative inverse scattering techniques.

\section{REFERENCES}

1. K. F. Warnick and W. C. Chew, "Numerical simulation methods for rough surface scattering," Waves Random Media 11, R1-R30 (2001).

2. A. Voronovich, Wave scattering from rough surfaces, vol. 17 (Springer Science \& Business Media, 2013).

3. M. Saillard and A. Sentenac, "Rigorous solutions for electromagnetic scattering from rough surfaces," Waves Random Media 11, 103-137 (2001).

4. J. Elson, J. Rahn, and J. Bennett, "Relationship of the total integrated scattering from multilayer-coated optics to angle of incidence, polarization, correlation length, and roughness cross-correlation properties," Appl. Opt. 22, 3207-3219 (1983).

5. D. Jordan and F. Moreno, "Enhanced backscattering and cross depolarization from multiscale surfaces," JOSA A 10, 1989-1995 (1993).

6. P. Hariharan, Optical interferometry (Academic press, 2003).

7. S. Kuwamura and I. Yamaguchi, "Wavelength scanning profilometry for real-time surface shape measurement," Appl. optics 36, 4473-4482 (1997).

8. P. de Groot, "Principles of interference microscopy for the measurement of surface topography," Adv. Opt. Photonics 7, 1-65 (2015).

9. R. Wombell and J. A. DeSanto, "The reconstruction of shallow roughsurface profiles from scattered field data," Inverse Probl. 7, L7 (1991).

10. I. Akduman, R. Kress, and A. Yapar, "Iterative reconstruction of dielectric rough surface profiles at fixed frequency," Inverse Probl. 22, 939 (2006).

11. Y.-Q. Jin and Z. Li, "Reconstruction of roughness profile of fractal surface from scattering measurement at grazing incidence," J. Appl. Phys. 89, 1922-1926 (2001).

12. Y.-Q. Jin, "Reconstruction of a heterogeneous fractal surface profile from scattering measurements at low grazing incidence," in "Antennas and Propagation Society International Symposium, 2005 IEEE," , vol. 3 (IEEE, 2005), vol. 3, pp. 445-448.

13. G. Bao and L. Zhang, "Shape reconstruction of the multi-scale rough surface from multi-frequency phaseless data," Inverse Probl. 32 , 085002 (2016).

14. M. Spivack, "Solution of the inverse-scattering problem for grazing incidence upon a rough surface," J. Opt. Soc. Am. A 9, 1352-1355 (1992).

15. M. Spivack, "Direct solution of the inverse problem for rough surface scattering at grazing incidence," J. Phys. A: Math. Gen. 25, 3295 (1992).

16. Z. Cai, D. Chen, and S. Lu, "Reconstruction of a fractal rough surface," Phys. D: Nonlinear Phenom. 213, 25-30 (2006).

17. C. D. Lines and S. N. Chandler-Wilde, "A time domain point source method for inverse scattering by rough surfaces," Computing. 75, 157180 (2005).

18. M. Cayoren, I. Akduman, A. Yapar, and L. Crocco, "Shape reconstruction of perfectly conducting targets from single-frequency multiview data," IEEE Geosci. Remote. Sens. Lett. 5, 383-386 (2008).

19. P. de Groot and L. Deck, "Surface profiling by analysis of white-light interferograms in the spatial frequency domain," J. modern optics $\mathbf{4 2}$ 389-401 (1995).

20. J. T. Johnson, R. J. Burkholder, J. V. Toporkov, D. R. Lyzenga, and W. J. Plant, "A numerical study of the retrieval of sea surface height profiles from low grazing angle radar data," IEEE Transactions on Geosci. Remote. Sens. 47, 1641-1650 (2009).

21. O. G. Nwogu and D. R. Lyzenga, "Surface-wavefield estimation from coherent marine radars," IEEE Geosci. Remote. Sens. Lett. 7, 631-635 (2010).

22. D. R. Lyzenga, O. G. Nwogu, R. F. Beck, A. O'Brien, J. Johnson, T. de Paolo, and E. Terrill, "Real-time estimation of ocean wave fields from marine radar data," in "Geoscience and Remote Sensing Symposium (IGARSS), 2015 IEEE International," (IEEE, 2015), pp. 36223625.

23. D. Schiavulli, F. Nunziata, G. Pugliano, and M. Migliaccio, "Reconstruction of the normalized radar cross section field from gnss-r delaydoppler map," IEEE J. Sel. Top. Appl. Earth Obs. Remote. Sens. 7, 1573-1583 (2014)

24. J. DeSanto, "Exact boundary integral equations for scattering of scalar 
waves from infinite rough interfaces," Wave Motion 47, 139-145 (2010).

25. F. D. Tappert, "The parabolic approximation method," in "Wave propagation and underwater acoustics," (Springer, 1977), pp. 224-287.

26. E. I. Thorsos, "Rough surface scattering using the parabolic wave equation," J. Acoust. Soc. Am. 82, S103-S103 (1987).

27. M. Spivack, "A numerical approach to rough-surface scattering by the parabolic equation method," J. Acoust. Soc. Am. 87, 1999-2004 (1990).

28. Z.-H. Gu, I. M. Fuks, and M. Ciftan, "Grazing angle enhanced backscattering from a dielectric film on a reflecting metal substrate," Opt. Eng. 43, 559-568 (2004)

29. D. S. Finley, P. Jelinsky, S. Bowyer, and R. F. Malina, "Design of the extreme ultraviolet explorer long-wavelength grazing incidence telescope optics," Appl. optics 27, 1476-1480 (1988).

30. G. Renaud, R. Lazzari, and F. Leroy, "Probing surface and interface morphology with grazing incidence small angle x-ray scattering," Surf. Sci. Reports 64, 255-380 (2009).

31. M. Spivack, J. Ogilvy, and C. Sillence, "Left-right splitting for electromagnetic scattering in 3d," IEE Proceedings-Science, Meas. Technol. 151, 464-466 (2004).

32. R. S. Awadallah and G. S. Brown, "Low-grazing angle scattering from rough surfaces in a duct formed by a linear-square refractive index profile," IEEE Transactions on Antennas Propag. 48, 1461-1474 (2000).

\section{APPENDIX A: CALCULATION OF EQUATION (13) WITH SINGULARITY}

Here, we give an approximation for the integral

$A(n, n)=\int_{x_{n-1}}^{x_{n}} \frac{1}{4 i} H_{0}^{(1)}\left(k\left|\left(x_{n}, h\left(x_{n}\right)\right)-\left(x^{\prime}, h\left(x^{\prime}\right)\right)\right|\right) \sqrt{\left.1+h^{\prime}\left(x^{\prime}\right)^{2}\right)} d x^{\prime}$

We first notice that

$$
H_{0}^{(1)}(\eta) \sim 1+\frac{2 i}{\pi}\left[\ln \left(\frac{\eta}{2}\right)+\gamma\right]+O\left(\eta^{2}\right),
$$

for small $\eta$, where $\gamma$ is the the Euler-Mascheroni constant. If changing of variable by

$$
\eta=k \sqrt{\left(x^{\prime}-x_{n}\right)^{2}+\left(h\left(x^{\prime}\right)-h\left(x_{n}\right)\right)^{2}},
$$

then

$$
d x^{\prime}=\frac{1}{k^{2}} \frac{\eta d \eta}{\left(x^{\prime}-x_{n}\right)+h^{\prime}\left(x^{\prime}\right)\left(h\left(x^{\prime}\right)-h\left(x_{n}\right)\right)} .
$$

Thus, the integral Eq. (28) with new variable Eq. (30) and approximation Eq. (29) becomes

$$
\begin{aligned}
& \int_{\eta_{0}}^{0} \frac{1}{4 i} H_{0}^{(1)}(k \eta) \frac{\sqrt{\left.1+h^{\prime}\left(x^{\prime}\right)^{2}\right)} \eta}{k^{2}\left[\left(x^{\prime}-x_{n}\right)+h^{\prime}\left(x^{\prime}\right)\left(h\left(x^{\prime}\right)-h\left(x_{n}\right)\right)\right]} d \eta \\
& \sim \int_{\eta_{0}}^{0} \frac{1}{4 i}\left[1+\frac{2 i}{\pi}\left(\ln \left(\frac{\eta}{2}\right)+\gamma\right)\right] W\left(x^{\prime}, x_{n}\right) d \eta,
\end{aligned}
$$

where $\eta_{0}=k \sqrt{\left(x_{n-1}-x_{n}\right)^{2}+\left(h\left(x_{n-1}\right)-h\left(x_{n}\right)\right)^{2}}$, and

$$
W\left(x^{\prime}, x_{n}\right)=\frac{\sqrt{\left.1+h^{\prime}\left(x^{\prime}\right)^{2}\right)}}{\left(x^{\prime}-x_{n}\right)+h^{\prime}\left(x^{\prime}\right)\left(h\left(x^{\prime}\right)-h\left(x_{n}\right)\right)},
$$

Define $\Delta x=x^{\prime}-x_{n}$ and $\Delta h=h\left(x^{\prime}\right)-h\left(x_{n}\right)$. Expanding $h$ we have

$$
h\left(x_{n}\right)=h\left(x^{\prime}\right)+h^{\prime}\left(x^{\prime}\right)\left(x_{n}-x^{\prime}\right),
$$

then $h\left(x^{\prime}\right) \Delta x=\Delta h$. As a result,

$$
W^{2}=\frac{1+h^{\prime}\left(x^{\prime}\right)^{2}}{\left(1+h^{\prime}\left(x^{\prime}\right)^{2}\right) \eta^{2}-\Delta h^{2}-h^{\prime} \Delta x^{2}+2 h^{\prime} \Delta x \Delta h} .
$$

but $-\Delta h^{2}-h^{\prime} \Delta x^{2}+2 h^{\prime} \Delta x \Delta h=-h^{\prime 2} \Delta x^{2}-h^{\prime} \Delta x^{2}+$ $2 h^{\prime} \Delta x h^{\prime} \Delta x=0$. Therefore, we get $W^{2}=1 / \eta^{2}$, and in fact $W=-1 / \eta$. Substitute this into the integral Eq. (31),

$$
\begin{aligned}
& A(n, n)=\frac{1}{k^{2}} \int_{0}^{\eta_{0}} \frac{1}{4 i}+\frac{1}{2 \pi} \ln \left(\frac{\eta}{2}\right)+\frac{1}{2 \pi} \gamma d \eta \\
& =\frac{1}{k^{2}}\left(\frac{\eta_{0}}{4 i}+\frac{1}{2 \pi} \gamma \eta_{0}\right)+\frac{1}{k^{2}} \int_{0}^{\eta_{0}} \frac{1}{2 \pi} \ln \left(\frac{\eta}{2}\right) d \eta .
\end{aligned}
$$

The last integral has no singularity

$$
\int_{0}^{\eta_{0}} \frac{1}{2 \pi} \ln \left(\frac{\eta}{2}\right) d \eta=\eta \ln \left(\frac{\eta}{2}\right)-\left.\eta\right|_{0} ^{\eta_{0}}=\eta_{0} \ln \left(\frac{\eta_{0}}{2}\right)-\eta_{0},
$$

since

$$
\lim _{\eta \rightarrow 0} \eta \ln \left(\frac{\eta}{2}\right)=\lim _{\eta \rightarrow 0} \frac{\ln \left(\frac{\eta}{2}\right)}{\frac{1}{\eta}}=\lim _{\eta \rightarrow 0} \frac{\frac{1}{\eta}}{-\frac{1}{\eta^{2}}}=\lim _{\eta \rightarrow 0}(-\eta)=0
$$

Finally, we have

$$
A(n, n)=\frac{1}{k^{2}}\left\{\frac{\eta_{0}}{4 i}+\frac{1}{2 \pi} \gamma \eta_{0}+\frac{1}{2 \pi} \eta_{0}\left[\ln \left(\frac{\eta_{0}}{2}\right)-1\right]\right\} .
$$

For another integral

$$
\begin{aligned}
& A(n, n+1)= \\
& \int_{x_{n}}^{x_{n+1}} \frac{1}{4 i} H_{0}^{(1)}\left(k\left|\left(x_{n}, h\left(x_{n}\right)\right)-\left(x^{\prime}, h\left(x^{\prime}\right)\right)\right|\right) \sqrt{\left.1+h^{\prime}\left(x^{\prime}\right)^{2}\right)} d x^{\prime},
\end{aligned}
$$

it can be obtained by symmetry. Therefore,

$$
\begin{aligned}
& \quad A(n, n+1)=\frac{1}{k^{2}}\left\{\frac{\eta_{0}}{4 i}+\frac{1}{2 \pi} \gamma \eta_{1}+\frac{1}{2 \pi} \eta_{1}\left[\ln \left(\frac{\eta_{1}}{2}\right)-1\right]\right\}, \\
& \text { where } \eta_{1}=k \sqrt{\left(x_{n+1}-x_{n}\right)^{2}+\left(h\left(x_{n+1}\right)-h\left(x_{n}\right)\right)^{2}} .
\end{aligned}
$$

\section{APPENDIX B: APPROXIMATION OF THE LAST SUBINTE- GRAL IN EQUATION (20)}

We approximate the subintegral with singularity

$$
\int_{x_{n-1}}^{x_{n}} \alpha \exp \left[\frac{i k\left(h\left(x_{n}\right)-h\left(x^{\prime}\right)\right)^{2}}{2\left(x_{n}-x^{\prime}\right)}\right] \frac{d x^{\prime}}{\sqrt{x_{n}-x^{\prime}}}
$$

Change of variable via

$$
\zeta=\sqrt{x_{n}-x^{\prime}}, \quad d x^{\prime}=-2 \zeta d \zeta .
$$

Apply the Taylor expansion on $h$ with $h\left(x^{\prime}\right)=h\left(x_{n}\right)=$ $h^{\prime}\left(x_{n}\right)\left(x^{\prime}-x_{n}\right)$, then we have

$$
\frac{\left(h\left(x_{n}\right)-h\left(x^{\prime}\right)\right)^{2}}{x_{n}-x^{\prime}}=\frac{h^{\prime}\left(x_{n}\right)^{2}\left(x_{n}-x^{\prime}\right)^{2}}{\left(x_{n}-x^{\prime}\right)}=h^{\prime}\left(x_{n}\right)^{2} \zeta^{2}
$$

The integral with new variable becomes

$$
\begin{aligned}
& \int_{\zeta_{0}}^{0} \alpha \exp \left[\frac{i k}{2} h^{\prime}\left(x_{n}\right)^{2} \zeta^{2}\right] \frac{1}{\zeta}(-2 \zeta) d \zeta \\
& =\int_{0}^{\zeta_{0}} 2 \alpha \exp \left[\frac{i k}{2} h^{\prime}\left(x_{n}\right)^{2} \zeta^{2}\right] d \zeta,
\end{aligned}
$$

where $\zeta_{0}=\sqrt{\delta}$ and $\delta$ is the spatial step-size $x_{n}-x_{n-1}$. For convenience we can write the above integral as

$$
2 \alpha \int_{0}^{\zeta_{0}} \exp \left[i \beta^{2} \zeta^{2}\right] d \zeta
$$

where $\beta=\sqrt{\frac{k}{2}}\left|h^{\prime}\left(x_{n}\right)\right|$ and then by using a change of variable $Z=\beta \zeta$, we can write the integral

$$
2 \frac{\alpha}{\beta} \int_{0}^{Z_{0}} \exp \left[i Z^{2}\right] d Z=2 \frac{\alpha}{\beta}\left[C\left(Z_{0}\right)+i S\left(Z_{0}\right)\right]
$$

where $Z_{0}=\beta \zeta_{0}$ and $C, S$ are the cosine and sin Fresnel integrals. 